\title{
Correction to: Early uranium mobilization in late Variscan strike-slip shear zones affecting leucogranites of central western Spain
}

\author{
F. J. López-Moro' ${ }^{1}$ - R. L. Romer ${ }^{2} \cdot$ D. Rhede ${ }^{2} \cdot$ A. Fernández ${ }^{1} \cdot$ S. M. Timón-Sánchez ${ }^{3} \cdot$ M. C. Moro $^{1}$
}

Published online: 6 November 2018

(c) Springer Nature Switzerland AG 2018

Correction to: Journal of Iberian Geology

https://doi.org/10.1007/s41513-018-0091-1

Due to an unfortunate oversight some mistakes occurred:

Paragraph 4.1. Uraninite chemical dating.

- at Deutsches GeoForschungs-Zentrum

- Multiple measurement of a homo-geneous

- a many individual crystal points on the crystal

Paragraph 6.2.1. Relation with shearing

(i) -Fried-rich et al. (1987)...

(ii) - episyenite (Table 1 or Fig. 6)

We apologize these mistakes.

The original article can be found online at https://doi.org/10.1007/ s41513-018-0091-1.

F. J. López-Moro

fjlopez@usal.es

1 Departamento de Geología, Facultad de Ciencias, Universidad de Salamanca, 37008 Salamanca, Spain

2 Deutsches GeoForschungsZentrum, Telegrafenberg, 14473 Potsdam, Germany

3 Instituto Geológico y Minero de España-IGME, Unidad de Salamanca, Plaza de la Constitución n ${ }^{\circ} 1$, planta $3^{\mathrm{a}}$, 37001 Salamanca, Spain 